\title{
SISTEMAS DE CÁLCULO COMO FORMAS DE LOGICISMO
}

\author{
Ángel Nepomuceno Fernández \\ Departamento de Filosofía y Lógica y Filosofía de la Ciencia \\ Universidad de Sevilla
}

\section{Introducción}

En la última década del siglo XX no parece tener vigencia alguna la filosofía de la lógica y la matemática conocida como logicismo, surgida a partir del pensamiento de Frege y alimentada por concepciones debidas a Russell, Wittgenstein, etc. Cabe pensar que la época de la preocupación por los fundamentos concluyó hace bastantes años; que hoy sólo se puede investigar en este campo desde la perspectiva del logicismo y éste, como se constata históricamente, no ha resistido el paso del tiempo, habiendo sucumbido ante una serie de resultados incontestablemente establecidos. Sin embargo, esto no es del todo exacto.

El logicismo puede ser considerado como un programa metafísico de investigación, según el cual, puesto que se concibe la matemática como derivable de la lógica, la matemática clásica sería reducida a lógica, en el sentido de que: a) "las nociones matemáticas sean definidas en términos de las nociones lógicas" y b) "los teoremas de la matemática sean demostrados como teoremas de la lógica". ${ }^{1}$ Los intentos por dar buen fin a dicho programa han resultado

${ }^{1}$ Kleene [8] p. 49. 
fallidos: tales han sido los casos de Grundgesetze y Principia Mathematica (y, en cierto sentido, New Foundations de Quine). Ahora bien, si distinguimos entre l) la idea de que la matemática, de alguna manera, es lógica y 2) la realización del programa en cuanto a la consecución de a) y b), entonces los fracasos afectan sobre todo a 2), mientras que 1) es más bien una actitud filosófica, compartida en principio por lógicos de diversas tendencias. $\mathrm{El}$ problema surgirá al tratar de aclarar el sentido de 1); por ejemplo, si se rechaza la lógica de orden superior al primero, dificilmente se admitirá a) y, en consecuencia, es imposible conseguir 2).

Un intento de revitalizar el logicismo consistirá en mantener 1) estableciendo un concepto de lógica a partir del cual actualizar 2). Otra manera es tratar de recuperar el planteamiento primitivo despojándolo de las nociones que provocaron su fracaso. En la segunda línea podemos situar la reconstrucción del logicismo fregeano propuesta por N.B. Cocchiarella. ${ }^{2}$ Una reconstrucción tal puede verse como un instrumento más adecuado para la tarea 2), la cual, por otra parte, ha de contar con los resultados limitativos aparecidos en el desarrollo de la lógica a partir de los años treinta. Pero si se presenta - como hace N.B. Cocchiarella - un cálculo modal de segundo orden que incorpora lo fundamental de la teoría de los tipos, el tratamiento intensional y extensional del concepto, cierta

2 Cocchiarella [1] y [2]. No entramos en sus planteamientos filosóficos en aras de obtener una lógica como teoría de la predicación que dé perfecta cuenta del papel del predicado en la lengua común. A este respecto, las ideas que desarrrolla tanto en [1] como [2] son coincidentes; el carácter logicista de los cálculos es más explícito en [2], de ahí que interese este trabajo particularmente. En cualquier caso, desde un punto de vista meramente formal, tanto en [1] como en [2] presenta cálculos similares cuya consideración es útil para nuestro propósito en estas páginas: cuestionar la validez de la afirmación según la cual la filosofía logicista no tiene vigencia alguna. 
doctrina de las clases, etc., éste sería una útil herramienta para abordar cuestiones de fundamentos que no pueden ser tachadas de "trasnochadas". En cuanto a su posible aplicación en computación e inteligencia artificial, se trata de una moderna utilidad adicional.

\section{Sistemas de cálculo de segundo orden}

Cualquiera que sea el modo de ejecutar la tarea 2), la reactualización de la doctrina logicista requiere una teoría general de la cuantificación, dado que la matemática clásica se concibe como reductible a lógica en el sentido a) y b), es decir, reductible a lógica de predicados. No se identifican plenamente lógica de predicados y teoría de conjuntos - como teoría general de la pertenencia- y ciertas porciones de la matemática no admiten la restricción de la cuantificación a términos individuales; así pues, ningún sistema de cálculo de predicados de primer orden representaría adecuadamente al logicismo.

Se definen unos sistemas de cálculo de segundo orden con identidad, lo que requiere una serie de definiciones.

Lenguaje formal. El lenguaje fornial $\mathfrak{L}$ consta de signos para variables (y constantes) individuales y predicativas de cualquier aridad, ${ }^{3}$ la constante diádica " $="$ ", los signos

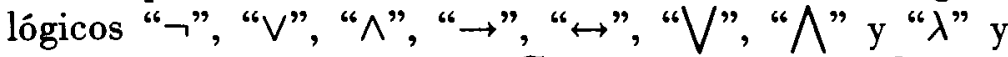
signos auxiliares. El conjunto $\mathfrak{F}$ de las fórmulas de $\mathfrak{L}$ viene definido por:

1) Si $R$ es un signo predicativo (variable o constante) $n$ ádico, para $n \geq 1$, y $b_{1}, b_{2}, \ldots, b_{n}$ son $n$ ocurrencias (no necesariamente distintas) de constantes o variables, entonces $R b_{1} b_{2} \ldots b_{n} \in \mathcal{F}$.

3 Cuando dos variables (o constantes) sean individuales o se trate de variables (o constantes) predicativas de la misma aridad, diremos que son del mismo tipo. 
2) Si $r$ y $s$ son variables (o constantes) del mismo tipo, entonces $r=s \in \mathcal{F}$.

3) $\mathrm{Si} \varphi \in \mathcal{F}$ y $x_{1}, x_{2}, \ldots, x_{n}$, para $n \geq 1$, son variables individuales distintas entre sí, entonces $\left(\lambda x_{1} x_{2} \ldots x_{n} \varphi\right)$ es un signo de predicado llamado $\lambda$-abstracto.

4) Si $\varphi \in \mathcal{F}, \neg \varphi \in \mathcal{F}$.

5) Si $\varphi, \psi \in \mathcal{F}$, entonces $\varphi \rightarrow \psi \in \mathcal{F}$.

6) $\mathrm{Si} \varphi \in \mathcal{F}$ y $s$ es una variable de cualquier tipo $\bigvee s \varphi \in \mathcal{F}$ y $\bigwedge s \varphi \in \mathcal{F}$.

Estratificación homogénea. Una fórmula $\varphi$ está homogéneamente estratificada si y sólo si:

1) Todas las subförmulas de $\varphi$ son de la forma $r=s$.

2) Si alguna subfórmula de $\varphi$ tiene la forma $\left(\lambda x_{1} x_{2} \ldots\right.$ $\left.x_{n} \psi\right) a_{1} a_{2} \ldots a_{n}$, entonces $a_{1}, a_{2}, \ldots, a_{n}$ son $n$ ocurrencias de constantes (o variables) individuales, no necesariamente distintas entre sí, para $n \geq 1$.

3) Si alguna subfórmula de $\varphi$ tiene la forma $R b_{1} b_{2} \ldots b_{n}$, para $n \geq 1$, entonces $b_{1}, b_{2}, \ldots, b_{n}$ son $n$ ocurrencias de constantes (o variables) individuales, no necesariamente distintas entre sí, para $n \geq 1$.

Un $\lambda$-abstracto $\left(\lambda x_{1} x_{2} \ldots x_{n} \psi\right)$, para cada $n \geq 1$, está homogéneamente estratificado si y sólo si la fórmula $\psi$ está homogéneamente estratificada.

Sistema de cálculo. Llamamos $\mathfrak{C}_{1}$ al cálculo que describimos a continuación. Son axiomas todas las fórmulas cuyos $\lambda$-abstractos $\rightarrow$ si los tuviere- están homogéneamente estratificados y sean tautologias o una de las siguientes formas:

1) $\bigwedge s(\varphi \rightarrow \psi) \rightarrow(\bigwedge s \varphi \rightarrow \bigwedge s \psi)$, donde $\varphi$ y $\psi$ son fórmulas y $s$ una variable de cualquier tipo. 
2) $\varphi \rightarrow \bigwedge s \varphi$, donde $\varphi$ es una fórmula y $s$ es una variable de cualquier tipo que no ocurre libre en $\varphi$.

3) $\bigvee s(r=s)$, donde $s$ es una variable del mismo tipo que $r$.

4) $a=b \rightarrow(\varphi \leftrightarrow \psi)$, donde $a$ y $b$ son del mismo tipo y $\psi$ se obtiene desde $\varphi$ por reemplazo de una o más ocurrencias libres de $b$ por ocurrencias libres de $a$.

5) $\left(\lambda x_{1} x_{2} \ldots x_{n} \varphi\right) a_{1} a_{2} \ldots a_{n} \leftrightarrow \varphi\left(a_{1} a_{2} \ldots a_{n} / x_{1} x_{2} \ldots x_{n}\right),{ }^{4}$ donde $x_{1}, x_{2}, \ldots, x_{n}$ son variables individuales distintas entre sí, $n \geq 1, \varphi$ es una fórmula $y$, para cada $i \leq n, a_{i}$ es una constante individual (o una variable individual para la que $x_{i}$ está libre en $\varphi$ ) y el $\lambda$-abstracto está homogéneamente estratificado.

6) $\left(\lambda x_{1} x_{2} \ldots x_{n} R x_{1} x_{2} \ldots x_{n}\right)=R$, donde $R$ es una variable (o constante) predicativa $n$-ádica, para $n \geq 1$, y $x_{1}, x_{2}, \ldots, x_{n}$ son variables individuales distintas entre sí.

7) $\bigvee F\left(\left(\lambda x_{1} x_{2} \ldots x_{n} \varphi\right)=F\right)$, donde $F$ es una variable predicativa $n$-ádica, para $n \geq 1$, la cual no ocurre libre en $\varphi$ y $x_{1}, x_{2}, \ldots, x_{n}$ son variables individuales distintas entre sí y el $\lambda$-abstracto está homogéneamente estratificado.

Las reglas del cálculo son: modus ponens y generalización, que podemos expresar, respectivamente, como Rl: de $\alpha \rightarrow \beta$ y $\alpha$ se infiere $\beta$ y R2: de $\alpha$ se infiere $\bigwedge s \alpha$.

Mediante $\mathcal{C}_{2}$ designamos el siguiente cálculo de segundo orden, tomado en parte del de A. Church, ${ }^{5}$ con el mismo lenguaje formal $\mathcal{L}$. $\mathcal{C}_{2}$ consta de los axiomas:

1) $\alpha \rightarrow(\beta \rightarrow \alpha)$.

2) $(\alpha \rightarrow(\beta \rightarrow \gamma)) \rightarrow((\alpha \rightarrow \beta) \rightarrow(\alpha \rightarrow \gamma))$.

3) $(\neg \beta \rightarrow \neg \alpha) \rightarrow(\alpha \rightarrow \beta)$.

${ }^{4} \varphi\left(a_{1} a_{2} \ldots a_{n} / x_{1} x_{2} \ldots x_{n}\right)$ es abreviatura de $S_{a_{1} a_{2} \ldots a_{n}}^{x_{1} x_{2} \ldots x_{n}} \varphi-$ noción de sustitución de A. Church [4] $\S 31$ y 51.

5 Ibid., p. 297. 
4) $\bigwedge s(\alpha \rightarrow \beta) \rightarrow(\alpha \rightarrow \bigwedge s \beta)$, siendo $s$ una variable de cualquier tipo que no ocurre libre en $\alpha$.

5) i) $\bigwedge x \alpha \rightarrow \alpha(a / x)$, donde $x$ es una variable de cualquier tipo y $a$ una variable (o constante) del mismo tipo que $x$;

ii) $\bigwedge p \alpha \rightarrow \alpha\left(\beta / / P x_{1} x_{2} \ldots x_{n}\right)$, donde $P$ es una variable predicativa $n$-ádica, para $n \geq 1$.

Las reglas de inferencia son las siguientes:

R1) Modus ponens.

R2) Generalización.

R3) Cambio de variable individual ligada. De $\alpha$, si $x$ es una variable individual que no ocurre libre en $\eta$ y $z$ es una variable individual que no ocurre en $\eta$, si $\alpha^{\prime}$ resulta de $\alpha$ al sustituir una ocurrencia particular de $\eta$ en $\alpha$ por $\eta^{\prime}$-resultante de sustituir en $\eta$ la ocurrencia ligada de $x$ por $z$-, entonces se infiere $\alpha^{\prime}$.

R4) Sustitución de variables individuales. De $\alpha$, si $x$ es una variable individual, si $y$ es una variable individual, y ninguna ocurrencia libre de $x$ en $\alpha$ ocurre en subfórmulas de la forma $\bigvee y \beta$, se infiere $\alpha(y / x)$.

Tiene además las siguientes definiciones:

Dl) $a=b==_{\text {def }} \bigwedge \mathrm{P}(P a \rightarrow P b)$, siendo $a$ y $b$ variables o constantes individuales.

D2) $R=S=$ def $\alpha \rightarrow \alpha^{\prime}$, siendo $R$ y $S$ variables (o constantes) predicativas de la misma aridad, $\alpha$ una fórmula cualquiera en la cual $R$ ocurre libre y $\alpha^{\prime}$ es la fórmula $\alpha(S / R)$.

Tanto para $\mathfrak{C}_{1}$ como para $\mathcal{C}_{2}$, podemos establecer definiciones sobre los signos de cuantificación: $\neg \bigwedge s \alpha={ }_{\text {def }}$ $\bigvee s \neg \alpha$, etc., que no especificamos por simplificar.

La adopción de estos sistemas en lugar de los origina- 
les, propuestos por Cocchiarella y Church, respectivamente, requiere una mínima aclaración. El cálculo estudiado por Church posee importantes propiedades metateóricas (corrección y completitud restringida, por ejemplo) que lo hacen sumamente interesante; el lenguaje formal del $\mathfrak{C}_{2}$ (el mismo que de $\mathcal{C}_{1}$ ), sin embargo, a diferencia del de Church, contiene $\lambda$-abstractos, pero es menos expresivo que el lenguaje formal usado por Cocchiarella, el cual admite que el signo $\lambda$ pueda llevar variables predicativas como sufijos y signos predicativos como argumentos (se trata del lenguaje $\mathfrak{L}^{\prime}$ que se menciona más abajo). Así pues, el uso de $\mathfrak{L}$ para ambos cálculos permite la comparación del sistema (ligeramente modificado) de Church con $\mathcal{C}_{1}$, punto de partida este último para obtener, tras algunas modificaciones, sistemas de cálculo logicistas.

\section{Proposición}

$\mathfrak{C}_{1}$ y $\mathcal{C}_{2}$ son equipotentes.

Para establecer la prueba asumimos el teorema de la deducción, según el cual si de $\Gamma, \alpha$ se infiere $\beta$, entonces de $\Gamma$ se infiere $\alpha \rightarrow \beta-\Gamma$ representa un conjunto de fórmulas-, siempre que $\beta$ no se obtenga por aplicación de las reglas $\mathrm{R} 2$ o R4 a $\alpha$ o a otra fórmula $\gamma$ de $\Gamma$ obtenida a su vez por aplicación de dichas reglas. Este teorema, también con restricciones, queda establecido para su mencionado cálculo por Church, cuyas reglas son las mismas que las de $\mathcal{C}_{2}$, en el que se demuestran las mismas tautologías que en $\mathfrak{C}_{1}$-axiomas de éste por definición-; asimismo, $R l$ y R2 son comunes a ambos cálculos. Por ello es fácilmente asumible el teorema en cuestión. También asumiremos el teorema de intercambio: si $\alpha$ es una fórmula que contiene $\alpha^{\prime}$ como subfórmula; si $\beta$ es la fórmula resultante de reemplazar alguna ocurrencia de $\alpha^{\prime}$ en $\alpha$ por otra fórmula $\beta^{\prime}$; entonces, si $\alpha^{\prime} \leftrightarrow \beta^{\prime}$ es demostrable, también lo es $\alpha \leftrightarrow \beta$. 
La prueba (para ambos cálculos) se haría considerando los casos en que $\alpha$ es cada uno de los axiomas y $\alpha^{\prime}$ una de las subfórmulas que aparece en la expresión de éstos.

1) $\Rightarrow$ ) Dado el cálculo $\mathcal{C}_{1}$, los axiomas 1), 2) y 3) de $\mathfrak{C}_{2}$ son tautologías.

Por lo que respecta al axioma 4) de $\mathcal{C}_{2}$, sea $\alpha$ una fórmula en la cual $s$ no ocurre libre; por axioma 1) de $\mathfrak{C}_{1}$

$$
\bigwedge s(\alpha \rightarrow \beta) \rightarrow(\bigwedge s \alpha \rightarrow \bigwedge s \beta)
$$

Pero $\bigwedge s \alpha$ es $\alpha$, por lo que ${ }^{6} \bigwedge s(\alpha \rightarrow \beta) \rightarrow(\alpha \rightarrow \bigwedge s \beta)$. Teniendo en cuenta que, en general, si $\alpha \leftrightarrow \beta$ entonces $\alpha \rightarrow \beta$, a partir del axioma 4) de $\mathcal{C}_{1}$ tendremos que $a=x \rightarrow(\alpha \leftrightarrow \alpha(a / x))$ y por tanto $a=x \rightarrow$ $(\alpha \rightarrow \alpha(a / x)$ ); por axioma 3$)$ de $\mathcal{C}_{1}$ y regla de modus ponens, $\alpha \rightarrow \alpha(a / x)$ y, por esta regla y generalización, $\bigwedge x(\alpha \rightarrow \alpha(a / x))$; por axioma 1) de $\mathcal{C}_{1}$ y aplicación de modus ponens, $\bigwedge x \alpha \rightarrow \bigwedge x \alpha(a / x)$; pero $\bigwedge x \alpha(a / x)$ es $\alpha(a / x)$, por lo que $\bigwedge x \alpha \rightarrow \alpha(a / x)$, expresión que corresponde al axioma 5) i) de $\mathcal{C}_{2}$. Por otra parte, de acuerdo con axioma 4) $\operatorname{de} \mathcal{C}_{1},\left(\lambda x_{1} x_{2} \ldots x_{m} \beta\right)=P \rightarrow\left(\alpha \leftrightarrow \alpha\left(\left(\lambda x_{1} x_{2} \ldots x_{m} \beta\right) / P\right)\right.$, para $m \geq 1$, por lo que

$$
\left(\lambda x_{1} x_{2} \ldots x_{m} \beta\right)=P \rightarrow\left(\alpha \rightarrow \alpha\left(\left(\lambda x_{1} x_{2} \ldots x_{m} \beta\right) / P\right),\right.
$$

siendo $\beta$ una fórmula cualquiera en la que $P$ no ocurre libre; teniendo en cuenta el axioma 7) de $\mathcal{C}_{1}$ y la regla de modus ponens,

$$
\alpha \rightarrow \alpha\left(\left(\lambda x_{1} x_{2} \ldots x_{m} \beta\right) / P\right) ;
$$

${ }^{6}$ Basta aplicar el teorema de intercambio. 
por generalización, $\bigwedge \mathrm{P}\left(\alpha \rightarrow \alpha\left(\left(\lambda x_{1} x_{2} \ldots x_{m} \beta\right) / P\right)\right.$; por axioma 1) de $\mathcal{C}_{1}$ y aplicación de modus ponens, $\bigwedge \mathrm{P} \alpha \rightarrow$ $\bigwedge \mathrm{P} \alpha\left(\left(\lambda x_{1} x_{2} \ldots x_{m} \beta\right) / P\right)$, pero $\bigwedge_{\mathrm{P} \alpha}\left(\left(\lambda x_{1} x_{2} \ldots x_{m} \beta\right) / P\right)$ es $\alpha\left(\left(\lambda x_{1} x_{2} \ldots x_{m} \beta\right) / P\right)$, por lo que $\bigwedge_{\mathrm{P}} \alpha \rightarrow \alpha\left(\left(\lambda x_{1} x_{2} \ldots\right.\right.$ $\left.\left.x_{m} \beta\right) / P\right)$; según el axioma 5) de $\mathfrak{C}_{1},\left(\lambda x_{1} x_{2} \ldots x_{m} \beta\right) a_{1} a_{2} \ldots$ $a_{m} \leftrightarrow \beta\left(a_{1}, a_{2}, \ldots, a_{m} / x_{1}, x_{2}, \ldots, x_{m}\right)$; por teorema de intercambio, $\alpha\left(\left(\lambda x_{1} x_{2} \ldots x_{m} \beta\right) / P\right) \leftrightarrow \alpha\left(\beta\left(a_{1}, a_{2}, \ldots\right.\right.$, $\left.\left.a_{m} / x_{1}, x_{2}, \ldots, x_{m}\right) /\left(\lambda x_{1} x_{2} \ldots x_{m} \beta\right) a_{1}, a_{2}, \ldots, a_{m}\right),{ }^{7}$ pero $\alpha\left(\beta\left(a_{1}, a_{2}, \ldots, a_{m} / x_{1}, x_{2}, \ldots, x_{m}\right) /\left(\lambda x_{1} x_{2} \ldots x_{m} \beta\right) a_{1}\right.$, $\left.a_{2}, \ldots, a_{m}\right)$ es $\alpha\left(\beta / / P x_{1} x_{2} \ldots x_{m}\right)$, por lo que $\bigwedge \mathrm{P} \alpha \rightarrow$ $\alpha\left(\beta / / P x_{1} x_{2} \ldots x_{m}\right)$, que expresa el axioma 5) ii) de $\mathcal{C}_{2}$.

Las reglas $R 1$ y $R 2$ de $\mathcal{C}_{2}$ son las mismas que en $\mathcal{C}_{1}$. En cuanto a R3 y R4, se pueden obtener a partir de asumir el intercambio.

2) $\Rightarrow$ ) A partir de los axiomas 1), 2) y 3) de $\mathcal{C}_{2}$, se pueden obtener todas las tautologías expresables en el lenguaje $\mathfrak{L}$ común a ambos cálculos.

Sea la hipótesis siguiente: $\bigwedge s(\varphi \rightarrow \psi)$, donde $s$ es una variable de cualquier tipo. Por axioma 5) i) $(\varphi \rightarrow \psi)$. De acuerdo con el mismo axioma 5) i), $\bigwedge s \varphi \rightarrow \varphi$. A partir de $\bigwedge s \varphi \rightarrow \varphi$ y $\varphi \rightarrow \psi$, suponiendo $\bigwedge s \varphi$, por Rl se obtiene $\varphi$ y, nuevamente por esta regla, $\psi$; por el teorema de la deducción, se obtiene finalmente $\bigwedge s \varphi \rightarrow \psi$. Por el teorema de la deducción, $\bigwedge s(\varphi \rightarrow \psi) \rightarrow(\bigwedge s \varphi \rightarrow \psi)$; de aquí, por axioma 4), $\bigwedge s(\varphi \rightarrow \psi) \rightarrow(\bigwedge s \varphi \rightarrow \bigwedge s \psi)$, expresión del axioma 1) de $\mathcal{C}_{1}$.

${ }^{7}$ Esta última fórmula obtenida de $\alpha\left(\left(\lambda x_{1} x_{2} \ldots x_{m} \beta\right) / P\right)$ reemplazando cada ocurrencia del $\lambda$-abstracto por la fórmula $\beta$, sustituyendo en ésta la $m$-pla de variables individuales por los argumentos de $P$.

${ }^{8}$ Church en [4] § 18, p. 109 y ss. establece la completitud de su cálculo proposicional. 
El axioma 2) de $\mathcal{C}_{1}$ se obtiene por teorema de la deducción a partir de $\varphi$ - con la variable (de cualquier tipo) $s$ libre- aplicando $\mathrm{R} 2$ de $\mathcal{C}_{2}$.

Según el axioma 3 ) de $\mathcal{C}_{2}$,

$$
(\neg \bigvee x(a=x) \rightarrow \neg(a=a)) \rightarrow(a=a \rightarrow \bigvee x(a=x))
$$

siendo $x$ una variable de cualquier tipo y $a$ una variable o constante del mismo tipo que $x$. Consideremos el caso en que son individuales; por axioma 5) de $\mathcal{C}_{2}, \bigwedge x \neg(a=x) \rightarrow$ $\neg(a=a)$, pero $\bigwedge x \neg(a=x)$ es $\neg \bigvee x(a=x)$, por lo que, aplicando Rl, $a=a \rightarrow \bigvee x(a=x)$. En general, supuesto $P a$ se puede obtener $P a$ y, por ello, $P a \rightarrow P a$ y de aquí, por aplicación de $\mathrm{R} 2$ de $\mathcal{C}_{2}, \bigwedge_{\mathrm{P}}(P a \rightarrow P a)$ o, lo que es lo mismo, $a=a$; aplicando $\mathrm{Rl}$ de $\mathcal{C}_{2}$ se obtiene $\bigvee x(a=x)$. Si $a$ y $x$ son signos predicativos (de la misma aridad), se procedería análogamente; se tendría que

$$
(\neg \bigvee x(a=x) \rightarrow \neg(\alpha \rightarrow \alpha)) \rightarrow((\alpha \rightarrow \alpha) \rightarrow \bigvee x(a=x))
$$

por axioma 5) de $\mathcal{C}_{2} \neg \bigvee x(a=x) \rightarrow \neg(a=a)$, puesto que $a$ es un signo predicativo; se obtiene, para alguna fórmula $\alpha$ tal que $a$ no está sometida a cuantificación, $\neg \bigvee x(a=$ $x) \rightarrow \neg(\alpha \rightarrow \alpha)$ y, por aplicación de $\mathrm{Rl}$ de $\mathcal{C}_{2},(\alpha \rightarrow \alpha) \rightarrow$ $\bigvee x(a=x)$; dado que se puede obtener, cualquiera que sea la fórmula $\alpha, \alpha \rightarrow \alpha$, aplicando nuevamente $\mathrm{Rl}$ de $\mathcal{C}_{2}$, $\bigvee x(a=x)$. En cualquier caso, pues, se obtiene la expresión del axioma 3 ) de $\mathcal{C}_{1}$. De acuerdo con la definición anterior de $\lambda$-abstracto, de manera semejante se obtiene el axioma 7) de $\mathcal{C}_{1}$, como una instancia del axioma 3 ) de $\mathcal{C}_{1}$ cuando el signo predicativo que se iguala a la variable ligada es un $\lambda$-abstracto. A partir del axioma 5) ii) de $\mathcal{C}_{2}$, teniendo 
en cuenta que $a=b$ es lo mismo que $\bigwedge \mathrm{P}(P a \rightarrow P b)$ y $\bigwedge \mathrm{P}(P b \rightarrow P a)$, se obtiene $a=b \rightarrow(\phi \leftrightarrow \psi)$, donde $\phi$ es una fórmula y $\psi$ es la fórrnula resultante de intercambiar en $\phi$ alguna ocurrencia libre de $b$ por $a$, que expresa el axioma 4) de $\mathcal{C}_{1}$.

Supuesto $\neg\left(\left(\lambda x_{1} x_{2} \ldots x_{n} P x_{1} x_{2} \ldots x_{n}\right)=P\right)$, por $\mathrm{R} 2 \mathrm{de}$ $\mathcal{C}_{2}$ y teorema de la deducción (teniendo en cuenta definición de cuantificadores), tenemos que

$$
\begin{gathered}
\neg\left(\left(\lambda x_{1} x_{2} \ldots x_{n} P x_{1} x_{2} \ldots x_{n}\right)=P\right) \rightarrow \\
\neg \bigvee \mathrm{P}\left(\left(\lambda x_{1} x_{2} \ldots x_{n} P x_{1} x_{2} \ldots x_{n}\right)=P\right) ;
\end{gathered}
$$

como por axioma 3$)$ de $\mathcal{C}_{2}\left(\neg\left(\left(\lambda x_{1} x_{2} \ldots x_{n} P x_{1} x_{2} \ldots x_{n}\right)=\right.\right.$ $\left.P) \rightarrow \neg \bigvee \mathrm{P}\left(\left(\lambda x_{1} x_{2} \ldots x_{n} P x_{1} x_{2} \ldots x_{n}\right)=P\right)\right) \rightarrow\left(\bigvee_{\mathrm{P}}\left(\left(\lambda x_{1} x_{2} \ldots\right.\right.\right.$ $\left.\left.\left.x_{n} P x_{1} x_{2} \ldots x_{n}\right)=P\right) \rightarrow\left(\left(\lambda x_{1} x_{2} \ldots x_{n} P x_{1} x_{2} \ldots x_{n}\right)=P\right)\right)$, aplicando $\mathrm{R} 1$ de $\mathcal{C}_{2}, \bigvee \mathrm{P}\left(\left(\lambda x_{1} x_{2} \ldots x_{n} P x_{1} x_{2} \ldots x_{n}\right)=P\right) \rightarrow$ $\left(\left(\lambda x_{1} x_{2} \ldots x_{n} P x_{1} x_{2} \ldots x_{n}\right)=P\right)$; como $\bigvee_{\mathrm{P}}\left(\left(\lambda x_{1} x_{2} \ldots\right.\right.$ $\left.x_{n} P x_{1} x_{2} \ldots x_{n}\right)=P$ ) es demostrable en $\mathcal{C}_{2}$, según se vio anteriormente, nuevamente por $\mathrm{Rl}$ de $\mathcal{C}_{2},\left(\lambda x_{1} x_{2} \ldots\right.$ $\left.x_{n} P x_{1} x_{2} \ldots x_{n}\right)=P$, que expresa el axioma 6) de $\mathcal{C}_{1}$.

De acuerdo con Henkin ${ }^{9}$ es demostrable en $\mathcal{C}_{2}$ el principio de comprehensión, es decir, $\bigvee \mathrm{P} \bigwedge x_{1} x_{2} \ldots x_{n}\left(P x_{1} x_{2} \ldots\right.$ $x_{n} \leftrightarrow \phi$ ), donde $P$ es una variable predicativa $n$-ádica, para $n \geq 1$, la cual no ocurre libre en $\phi$, es demostrable en $\mathfrak{C}_{2}$. Sea $P$-ádico tal que $\bigwedge x_{1} x_{2} \ldots x_{n}\left(P x_{1} x_{2} \ldots x_{n} \leftrightarrow \phi\right)$; teniendo en cuenta el axioma 4) y aplicando Rl -ambos de $\mathcal{C}_{2}$-, para las $n$ ocurrencias de signos individuales $a_{1}, a_{2}, \ldots, a_{n}$, se obtiene $\left(P a_{1} a_{2} \ldots a_{n} \leftrightarrow \phi\left(a_{1} a_{2} \ldots\right.\right.$ $\left.a_{n}\left(x_{1} x_{2} \ldots x_{n}\right)\right)$; dado que en $\mathcal{C}_{2}$ se puede demostrar $\left(\lambda x_{1} x_{2} \ldots x_{n} P x_{1} x_{2} \ldots x_{n}\right)=P$, por aplicación del teorema de intercambio se obtiene $\left(\lambda x_{1} x_{2} \ldots x_{n} P x_{1} x_{2} \ldots x_{n}\right) a_{1} a_{2} \ldots$

${ }^{9}$ L. Henkin [6] p. 203. 
$a_{n} \leftrightarrow \phi\left(a_{1} a_{2} \ldots a_{n} / x_{1} x_{2} \ldots x_{n}\right)$, que expresa el axioma 5) de $\mathcal{C}_{1}$.

Las reglas de modus ponens y generalización de $\mathcal{C}_{1}$ no son más que las reglas $R 1$ y $R 2$, respectivamente, de $\mathcal{C}_{2}{ }^{10}$

\section{Cálculos ampliados}

Sea la siguiente formulación del principio de extensionalidad -al que abreviadamente nos referiremos como "(Ext)"-:

$\bigwedge x_{1} x_{2} \ldots x_{n}(\phi \leftrightarrow \psi) \rightarrow\left(\lambda x_{1} x_{2} \ldots x_{n} \phi\right)=\left(\lambda x_{1} x_{2} \ldots x_{n} \psi\right)$, donde $x_{i}$, para cada $i \leq n$, es una variable individual y $\phi$ y $\psi$ son fórmulas.

10 El cálculo que Cocchiarella [2] propone como similar al de Grundgesetze, en el sentido de contar con los mismos axiomas -0 sus equivalentes - pero eliminando la causa de la contradicción hallada por Russell, no coincide exactamente con $\mathcal{C}_{1}$, de acuerdo con la explicación dada más arriba. Por otra parte, Cocchiarella pretende obtener un cálculo que exprese lo que èl denomina primer logicismo de Russéll, el cual correspondería a los planteamientos russellianos previos a Principia Mathematica. Por este motivo, apelando a un modo de entender el concepto intensionalmente, que él atribuye al propio Russell, propone un cálculo intensional; éste sería una forma intensional del logicismo de Frege y del primer logicismo de Russell. De esta manera - concluye Cocchiarella [2] p. 249 "logicism, whether in Frege's or Russell's early form, is alive and well". La circunstancia de que $\mathfrak{C}_{2}$ pueda ser más elegante que $\mathcal{C}_{1}$ no modifica el planteamiento inicial, aunque en Cocchiarella [1] aparece una defensa de los cálculos de éste frente a los sistemas quineanos de $N F$ y $M L$ (New Foundations y Mathematical Logic, respectivamente) tanto por elegancia como por erradicación de las controversias suscitadas por éstos.

En lo que sigue procedemos a la ampliación del cálculo a partir de $\mathcal{C}_{1}$, aunque no nos planteamos tanto obtener un sistema genuino representante del logicismo cuanto constatar que un cálculo logicista pueda resultar de utilidad; que éste sea más fregeano que russelliano es otro problema (interesante sin duda y que preocupa a Cocchiarella, lo que lo lleva a especular sobre si las ampliaciones recogen un platonismo conceptual, una disquisición sobre la tesis de Abelardo, etc., pero no son pertinentes en este contexto). 
$\mathcal{C}_{1}+($ Ext $)$, al que designamos mediante $\mathcal{C}_{3}$, es el cálculo resultante de añadir (Ext) a $\mathcal{C}_{1}$.

A continuación presentamos otro sistema de cálculo al que designamos mediante $\mathcal{C}_{4}$ que será, como veremos más abajo, de al menos la misma potencia deductiva que $\mathcal{C}_{1}$, partiendo del mismo lenguaje formal $\mathfrak{L}_{\mathcal{C}_{4}}$ tiene las mismas reglas que $\mathcal{C}_{1}$ y los siguientes axiomas, además de todas las fórmulas que sean tautologías y sus $\lambda$-abstractos estén homogéneamente estratificados:

1) $\bigwedge s(\phi \rightarrow \psi) \rightarrow(\bigwedge s \phi \rightarrow \bigwedge s \psi)$, donde $\phi$ y $\psi$ son fórmulas y $s$ una variable de cualquier tipo.

2) $\phi \rightarrow \bigwedge s \phi$, donde $\phi$ es una fórmula y $s$ una variable de cualquier tipo.

3) $\bigwedge x \bigvee y(x=y)$, donde $x$ es una variable de cualquier tipo y $y$ es una variable del mismo tipo que $x$.

4) $a=a$, donde $a$ es una constante de cualquier tipo.

5) $(a=b) \rightarrow(\phi \leftrightarrow \psi)$, donde $a$ es una variable (o constante) de cualquier tipo, $b$ es una variable (o constante) del mismo tipo que $a$ y $\psi$ es la fórmula obtenida de $\phi$ por reemplazo de alguna ocurrencia libre de $a$ por $b$.

6) $\left(\lambda x_{1} x_{2} \ldots x_{n} P x_{1} x_{2} \ldots x_{n}\right)=P$, donde $P$ es una variable predicativa $n$-ádica, para $n \geq 1$, y $x_{1}, x_{2}, \ldots, x_{n}$ son variables individuales.

7) $\left(\lambda x_{1} x_{2} \ldots x_{n} \phi\right) a_{1} a_{2} \ldots a_{n} \leftrightarrow \bigvee x_{1} x_{2} \ldots x_{n}\left(a_{1}=x_{1} \wedge\right.$ $\left.a_{2}=x_{2} \wedge \ldots \wedge a_{n}=x_{n} \wedge \phi\right)$, donde, para cada $i \leq n, x_{i}$ es una variable individual y $a_{i}$ es una constante individual.

8) $\bigvee F\left(\left(\lambda x_{1} x_{2} \ldots x_{n} \phi\right)=F\right)$, donde $F$ es una variable predicativa $n$-ádica, para $n \geq 1 \mathrm{y}$, para cada $i \leq n, x_{i}$ es una variable individual.

9) $\left(\bigvee y\left(a_{1}=y\right) \wedge \bigvee y\left(a_{2}=y\right) \wedge \ldots \wedge \bigvee y\left(a_{k}=y\right)\right) \rightarrow$ $\bigvee F\left(\left(\lambda x_{1} x_{2} \ldots x_{n} \phi\right)=F\right)$, donde, para $k \geq 1$ y $n \geq 1$, 
$a_{1}, a_{2}, \ldots, a_{k}$, son constantes individuales, $x_{1}, x_{2}, \ldots, x_{n}$ -y las $y$ de la subfórmula antecedente- son variables individuales y $F$ es una variable predicativa $n$-ádica.

Se trata de que en $\mathcal{C}_{4}$ se capten, al menos, las mismas ideas que en $\mathcal{C}_{1}$, correspondientes a concepciones logicistas. Para reducir los presupuestos existenciales, se cambian los axiomas 3) y 5) de $\mathcal{C}_{l}$, y en su lugar se adoptan los que aparecen en $\mathcal{C}_{4}$ como 3 ) y 7 ), respectivamente; los axiomas 4) y 9) de $\mathcal{C}_{4}$ son especificos de este cálculo.

Para comprobar que $\mathcal{C}_{4}$ tiene al menos la misma potencia deductiva que $\mathcal{C}_{1}$, hay que probar que los axiomas 3 ) y 5 ) de $\mathcal{C}_{1}$ son demostrables en $\mathcal{C}_{4}$. Consideremos en primer lugar que una expresión de la forma $\bigwedge x \alpha \rightarrow \alpha(a / x)$, siendo $x$ una variable de cualquier tipo y $a$ una variable del mismo tipo que $x$, es demostrable en $\mathcal{C}_{4}$. En efecto, más arriba se estableció que tal expresión era demostrable en $\mathcal{C}_{1}$, apelando a axiomas que figuran asimismo como axiomas de $\mathcal{C}_{4}$, por lo que es demostrable en $\mathcal{C}_{4}$. Sea entonces la siguiente expresión del axioma 4) de $\mathcal{C}_{4}: \wedge y \bigvee x(y=x)$, donde $x$ y $y$ son variables del mismo tipo; de aquí se obtiene $\bigvee x(a=x)$, expresión del axioma 3$)$ de $\mathcal{C}_{1}$.

Por otra parte, supuesto $\left(\lambda x_{1} x_{2} \ldots x_{n} \phi\right) a_{1} a_{2} \ldots a_{n}$, por axioma 7) de $\mathcal{C}_{4}$ y aplicación de modus ponens, se obtiene

$$
\bigvee x_{1} x_{2} \ldots x_{n}\left(a_{1}=x_{1} \wedge a_{2}=x_{2} \wedge \ldots \wedge a_{n}=x_{n} \wedge \phi\right) ;
$$

teniendo en cuenta axioma 3 ) de $\mathcal{C}_{4}$, eliminación de $\Lambda$ - demostrable en $\mathcal{C}_{4}$, como se acaba de ver-, y siendo $b_{1}, b_{2}, \ldots, b_{n}$ tales que, para cada $i \leq n, a_{i}=b_{i}$, se obtiene

$$
a_{1}=b_{1} \wedge a_{2}=b_{2} \wedge \ldots \wedge a_{n}=b_{n} \wedge \phi\left(b_{1} b_{2} \ldots b_{n} / x_{1} x_{2} \ldots x_{n}\right)
$$

y de aquí (dado que $\alpha_{1} \wedge \alpha_{2} \wedge . . \wedge \alpha_{m} \rightarrow \alpha_{i}$, para todo $m \geq$ 1 e $i \leq m$, es una tautología), $\phi\left(b_{1} b_{2} \ldots b_{n} / x_{1} x_{2} \ldots x_{n}\right)$; teniendo en cuenta axioma 5 ) de $\mathcal{C}_{4}$ y aplicación sucesiva de 
modus ponens, se obtiene la fórmula equivalente $\phi\left(a_{1} a_{2} \ldots\right.$ $\left.a_{n} / x_{1} x_{2} \ldots x_{n}\right)$. A partir de esta fórmula, teniendo en cuenta los axiomas 3) y 4) de $\mathfrak{C}_{4}$, se puede obtener la fórmula $a_{1}=b_{1} \wedge a_{2}=b_{2} \wedge \ldots \wedge a_{n}=b_{n} \wedge \phi\left(b_{1} b_{2} \ldots b_{n} / x_{1} x_{2} \ldots x_{n}\right)$ que, de acuerdo con el axioma 7) de $\mathcal{C}_{4}$, es equivalente a $\left.\lambda x_{1} x_{2} \ldots x_{n} \phi\right) a_{1} a_{2} \ldots a_{n}$. Así pues, el axioma 5) de $\mathcal{C}_{1}$ es demostrable en $\mathcal{C}_{4}$.

Los cálculos $\mathfrak{C}_{1}$ y $\mathfrak{C}_{4}$ pueden ampliarse -aumentando con ello su potencia deductiva respecto de $\mathfrak{C}_{2}$ - introduciendo las siguientes modificaciones. El lenguaje $\mathfrak{L}$ se modifica por lo que respecta a la cláusula 3 ) usada para definir las fórmulas; en su lugar se establece:

$\left.3^{\prime}\right)$ Si $\varphi$ es una fórmula y $x_{1}, x_{2}, \ldots, x_{n}$, para $n \geq 1$, son $n$ variables de cualquier tipo distintas entre sí, entonces $\left(\lambda x_{1} x_{2} \ldots x_{n} \varphi\right)$ es un predicado $n$-ádico.

Por lo que respecta a la cláusula 2) de la definición de estratificación homogénea, se sustituye por la cláusula:

$\left.2^{\prime}\right)$ Si alguna subfórmula de $\varphi$ tiene la forma $\left(\lambda x_{1} x_{2} \ldots\right.$ $\left.x_{n} \psi\right) a_{1} a_{2} \ldots a_{n}$, para $n \geq 1$, entonces $a_{1}, a_{2}, \ldots, a_{n}$, son $n$ ocurrencias (no necesariamente distintas) de variables o constantes tales que, para cada $i \leq n, a_{i}$ es del mismo tipo que $x_{i}$.

Sea $\mathscr{L}^{\prime}$ el lenguaje modificado, el cual posee mayor capacidad expresiva que el anterior y permitirá mejor representación de concepciones logicistas; como ejemplo de ello tenemos la posibilidad de representar en tal lenguaje la nominalización de predicados: el significado de un signo predicativo como concepto o como objeto (extensión de concepto) en el contexto de las fórmulas en que ocurre, a saber, concepto cuando está en posición de predicado y objeto en la de argumento. Dado lo específico de $\mathfrak{L}^{\prime}$, una teoría de los tipos - útil para eludir la paradoja de Russell-- es formulable como sistema formal de segundo 
orden que tiene $\mathfrak{L}^{\prime}$ como lenguaje. Mediante $\mathcal{C}_{1}^{*}$ designamos el cálculo que contiene los mismos axiomas y reglas que $\mathcal{C}_{1}$, si bien haciendo uso del lenguaje $\mathfrak{L}^{\prime}$, tomando la noción modificada de estratificación homogénea y exigiendo que las fórmulas mencionadas en los axiomas sean homogéneamente estratificadas. Análogamente, $\mathcal{C}_{4}^{*}$ denotará el cálculo obtenido por la misma modificación relativa a $\mathfrak{C}_{4}$. De la misma manera, podemos exigir que las fórmulas que aparecen en la expresión de (Ext) - siendo fórmulas de $\mathfrak{L}^{\prime}$ estén homogéneamente estratificadas, en cuyo caso se designará mediante $\left(\right.$ Ext $\left.^{*}\right)$. De una modificación de $\mathcal{C}_{1}+($ Ext) resulta $\mathcal{C}_{1}^{*}+\left(\mathrm{Ext}^{*}\right)$, por lo que se le puede denominar $\mathcal{C}_{3}^{*}$.

El cálculo $\mathcal{C}_{3}$ es de mayor potencia deductiva que $\mathfrak{C}_{1}$, siendo, por tanto, de mayor potencia deductiva que $\mathfrak{C}_{2}$. Como hemos visto, $\mathfrak{C}_{4}$ tiene al menos la misma potencia que $\mathcal{C}_{1}$ por lo que $\mathcal{C}_{4}^{*}$ tendrá al menos la misma potencia deductiva que $\mathcal{C}_{1}^{*}$, pero no es así con respecto a $\mathcal{C}_{3}^{*}$. Si consideramos el cálculo $\mathcal{C}_{4}^{*}+\left(\mathrm{Ext}^{*}\right)$-al que nos referiremos mediante $\mathcal{C}_{5}^{*}$-, podemos afirmar que $\mathcal{C}_{5}^{*}$ tendrá al menos la misma potencia deductiva que $\mathcal{C}_{3}^{*}$. Como es evidente, $\mathcal{C}_{3}^{*}$ y $\mathcal{C}_{5}^{*}$ son de mayor potencia deductiva que $\mathcal{C}_{2}$.

Cocchiarella ${ }^{11}$ establece la equiconsistencia de $\mathcal{C}_{3}^{*}$ con la versión de $N F$ de Quine modificada por Jensen [7]; asimismo, que si $\mathcal{C}_{3}^{*}$ es consistente, entonces $\mathcal{C}_{5}^{*}$ es tambiên consistente, es decir, que si $N F$ modificado es consistente, entonces $\mathcal{C}_{5}^{*}$ es consistente; estos resultados los extiende a los sistemas que presentamos en el siguiente apartado. Una tarea de interés - que no abordamos dado el objeto del presente trabajo- es investigar la corrección de estos cálculos.

La definibilidad del número natural se facilita con estos sistemas - como algo que corresponde a un concepto, en términos de nominalización y expresión y obtención de $\lambda$ -

11 [I] Pp. 230 y 242. 
abstractos. Por otra parte, no se presupone una colección infinita de objetos concretos, no aparece un axioma de infinitud y, sin embargo, sus axiomas -en particular los de $\mathcal{C}_{5}^{*}$ (como los de $\mathcal{C}_{4}$ ) - garantizan infinitos, en el sentido de que sea infinito el número total de objetos, es decir, el total de objetos concretos y de $\lambda$-abstractos. Estas razones, además de las mencionadas más arriba, se pueden aducir a favor de los intentos de una reconstrucción logicista.

\section{Sistemas intensionales}

A partir de $\mathcal{C}_{1}^{*}$ y $\mathcal{C}_{4}^{*} \longrightarrow \mathcal{C}_{3}^{*}$ y $\mathcal{C}_{5}^{*}$, respectivamente, pues se alcanzan lo que podemos denominar versiones intensionales de éstos- vamos a obtener sendos cálculos intensionales, procediendo como se explica a continuación.

$\mathcal{L}^{\prime}$ se modifica en el sentido de contener entre sus signos lógicos el operador " $\square$ ". No hacemos uso de " $\diamond$ " dado que $\diamond \varphi$, para cualquier fórmula $\varphi$, se puede definir como $\neg \square \neg \varphi$. En la definición de las fórmulas de $\mathfrak{L}^{\prime}$ hay que añadir la siguiente cláusula: si $\varphi$ es una fórmula, $\square \varphi$ es una fórmula.

$\square \mathcal{C}_{3}^{*}$ es el cálculo que consta de todos los axiomas y reglas de $\mathcal{C}_{1}^{*}$, además de los esquemas axiomáticos del cálculo modal S5, la regla de la generalización modal -según la cual, para cada fórmula $\varphi$, si $\varphi$ es demostrable en el cálculo, entonces $\square \varphi$ también es demostrable-, y el principio de intensionalidad, que se puede expresar como

$\square \bigwedge x_{1} x_{2} \ldots x_{n}(\varphi \leftrightarrow \psi) \rightarrow\left(\lambda x_{1} x_{2} \ldots x_{n} \varphi\right)=\left(\lambda x_{1} x_{2} \ldots x_{n} \psi\right)$

y al que nos referiremos mediante $\left(\square \mathrm{Ext}{ }^{*}\right)$ por su semejanza formal con $\left(\mathrm{Ext}^{*}\right)$. Añadiendo a $\mathcal{C}_{4}^{*}$ los esquemas axiomáticos de S5, la regla de generalización modal y ( $\left.\square \mathrm{Ext}^{*}\right)$, se obtiene otro cálculo que designaremos mediante $\square \mathcal{C}_{5}^{*}$. 
Estos cálculos podrían definirse incorporando los esquemas axiomáticos del cálculo modal $\mathrm{S} 4$ en lugar de los de S5, pero ello no modifica en lo esencial el sentido de este párrafo: la circunstancia de que se pueda presentar una forma intensional de cálculo logicista. En cualquier caso, S4 tiene más aplicación en programación que S5. ${ }^{12}$

$\square \mathcal{C}_{3}^{*}$ y $\square \mathcal{C}_{5}^{*}$ son sistemas de cálculo que pueden calificarse de logicistas a tenor de que han sido elaborados teniendo en cuenta concepciones logicistas. En concreto el sistema de Grundgesetze está integrado nor una serie de axiomas demostrables en estos cálculos, a excepción del conocido como Ley $V$, a partir del cual surge la paradoja de Russell. En efecto, las leyes de Grundgesetze, expresadas mediante $\mathfrak{L}^{\prime}$ y sin tener en cuenta la noción de estratificación, son las siguientes:

I) $\varphi \rightarrow(\psi \rightarrow \varphi)$, donde $\varphi$ y $\psi$ son förmulas cualesquiera.

II) a) $\bigwedge x \alpha \rightarrow \alpha(a / x)$, donde $\alpha$ es una fórmula, $x$ una variable individual y $a$ una variable (o constante) individual.

b) $\bigwedge \mathrm{P} \alpha \rightarrow \alpha\left(\beta / / P x_{1} x_{2} \ldots x_{n}\right)$, donde $\alpha$ y $\beta$ son fórmulas y $P$ es una variable predicativa $n$-ádica, para $n \geq 1$.

III) $a=b \rightarrow(\phi \rightarrow \psi)$, donde $a$ y $b$ son del mismo tipo y $\psi$ es la fórmula resultante de $\phi$ por reemplazo de alguna ocurrencia libre de $a$ por $b$.

IV) $\neg(\phi \leftrightarrow \neg \psi) \rightarrow(\phi \leftrightarrow \psi)$, donde $\phi$ y $\psi$ son fórmulas cualesquiera.

V) $\left(\lambda x_{1} x_{2} \ldots x_{n} \varphi\right)=\left(\lambda x_{1} x_{2} \ldots x_{n} \psi\right) \leftrightarrow \bigwedge x_{1} x_{2} \ldots x_{n}(\varphi \leftrightarrow$ $\psi$ ), donde, para $n \geq 1$, para todo $i \leq n$, $x_{i}$ es una variable individual y $\varphi$ у $\psi$ son fórmulas.

VI) $F=\left(\lambda x_{1} x_{2} \ldots x_{n} F x_{1} x_{2} \ldots x_{n}\right)$, donde $F$ es un signo predicativo $n$-ádico, para $n \geq 1$.

12 Vid. J. Cuena [3] p. 277. 
Las reglas son las de modus ponens y generalización.

I) y III) son expresiones tautológicas por lo que forman parte de $\square \mathcal{C}_{3}^{*}$. II) a) y II) b) se pueden demostrar en $\square \mathfrak{C}_{3}^{*}$, como se ha visto más arriba al comparar $\mathcal{C}_{1}$ con $\mathcal{C}_{2}$ y $\mathcal{C}_{1}$ con $\mathcal{C}_{4}$. III) resulta inmediatamente del axioma 4) de $\mathcal{C}_{1}$ y VI) es exactamente el axioma 6) de $\mathcal{C}_{1} . \mathrm{V}$ ), en un único sentido de la coimplicación, corresponde a (Ext). Como es obvio, no podemos decir que $\square \mathcal{C}_{3}^{*}-\mathrm{y}$ tampoco $\square \mathcal{C}_{5}^{*}$ - sea de al menos la misma potencia deductiva que el cálculo de Grundgesetze, puesto que este último es inconsistente. No obstante, si se modifica Grundgesetze, cambiando su ley V) por (Ext) y adoptando la noción de estratificación, entonces los teoremas de este Grundgesetze modificado serán teoremas de $\square \mathcal{C}_{3}^{*}$ y, en consecuencia, de $\square \mathcal{C}_{5}^{*}$. En este sentido, pues, tanto $\square \mathcal{C}_{3}^{*}$ como $\square \mathcal{C}_{5}^{*}$ son cálculos logicistas.

Pero estos cálculos - si son consistentes - no arrastran, por así decirlo, el lastre del planteamiento de Frege, que llevaba implícita la paradoja de Russell. Las restricciones impuestas, como la estratificación homogénea y la limitación de la ley $V$ a ( $\square \mathrm{Ext}^{*}$ ), impiden derivar dicha paradoja. Una ventaja añadida es que se trate de cálculos intensionales, cuyo estudio y desarrollo está cada vez más en boga; por ello, una revisión semejante a la propuesta por Cocchiarella podría revitalizar el logicismo.

Otro aspecto de este tipo de reconstrucciones es la mejora del aparato conceptual con el que se estudia la corriente de la filosofía de la lógica que nos ocupa. El mayor o menor grado de interés para la historia de la lógica no dice nada acerca de la vigencia o no de aquella doctrina. A este respecto, y teniendo en cuenta $\square \mathcal{C}_{3}^{*}$ y $\square \mathcal{C}_{5}^{*}$, los problemas que atañen a la ejecución de la tarea 2), mencionada más arriba, no desaparecen, pero el sentido de 1) queda más explícito. 


\section{BIBLIOGRAFÍA}

[1] Cncchiarella, N.B., "Two $\lambda$-extensions of the Theory of Homogeneous Simple Types as a Second-Order Logic", Notre Dame Journal of Formal Logic, vol. 26, no. 4, 1985, pp. 377407.

[2] —_, "Frege, Russell and Logicism", en L. Haaparanta y J. Hintikka (comps.), Frege Synthesized, D. Reidel Publishing Company, Dordrecht, 1986, vol. 181, pp. 197-252.

[3] Cuena, J., Lógica informática, Alianza, Madrid, 1985.

[4] Church, A., Introduction to Mathematical Logic, 6a. impresión, Princeton University Press, Princeton, 1970.

[5] Frege, G., Conceptografía. Los fundamentos de la aritmética. Otros estudios filosóficos, trad. H. Padilla, Instituto de Investigaciones Filosóficas-UNAM, México, 1972.

[6] Henkin, L., "Banishing the Rule of Substitution for Functional Variables", The Journal of Symbolic Logic, vol. 18, no. 3, 1953, pp. 201-208.

[7] Jensen, R.B., "On the Consistency of a Slight (?) Modifications of Quine's, New Foundations", en D. Davidson y H. Hintikka (comps.), Words and objections. Essays on the Work of W.V. Quine, D. Reidel Publishing Company, Dordrecht, 1975, vol. 21, pp. 278-29l.

[8] Kleene, S.C., Introducción a la metamatemática, trad. M. Garrido, Tecnos, Madrid, 1974. 


\section{SUMMARY}

The logicism may be regarded like a fossil stone that has not utility nowadays. In this sense, logicism took care of the research about the foundations of mathematics but apparently its task arrived at its end many years ago because of some results that were established during the century. However it is not wholly right. Understanding logicism as an attempt to reduce classical mathematics to logic means we can distinguish: 1) the idea according to which mathematic is logic in some way, and 2) a inetaphysical program of research to: a) define mathematical notions as logical notions, and b) show that the mathematical theorems are logical theorems.

The failure (if so) concerned to 2), since 1) was assumed by many logicians. Recovering logicism is not easy and there may be several ways. One of them is the one followed by N.B. Cocchiarella whose systems (there are more than one) represent a form of logicism (Frege's or Russell's form). From those systems -though a bit changed from my own point of view- we can define a modal calcule that may have application in computer science, what would not be a stale work.

From a common language we take in account iwo systems in order to show that Cocchiarella's modified system is as powerful deductively as that of Church modified functional second order calcule. We can obtain new systems that represent form of logicism and are more powerful than that of Church enlarging Cochiarella's modified system. These new systems, that becomes modal systems provided that one adds appropiate modal tools (then they may be used in computer science), may be useful to study logicism itself (as historical philosophy of logic and mathematics). 